Communications in Physics, Vol.21, No. 4 (2011), pp. 359-364

\title{
POLARIZATION LABELLING SPECTROSCOPY FOR NaLi
}

\author{
NGUYEN TIEN DUNG, DINH XUAN KHOA, AND NGUYEN HUY BANG \\ Vinh University \\ PHAM VAN TRONG \\ Hong Duc University
}

\begin{abstract}
We use the polarization labelling spectroscopy technique for simplification of the spectra of alkali-metal NaLi molecule. As a result, the rotational resolution observation for NaLi in the $2^{1} \Pi$ electronic state is reported for the first time.
\end{abstract}

\section{INTRODUCTION}

The alkali-metal diatomic molecules are very attractive for both theoreticians and experimentalists because they have a relatively simple electronic structure. Their electronic structure is frequently considered by theoreticians as a very convenient model for introducing approximations which can be further applied to more complex molecular systems. From the experimental point of view, alkali-metal diatomic molecules of their main absorption bands in the visible and UV regions are very convenient objects for investigations with modern laser spectroscopy techniques. Investigations of alkali-metal molecules have recently given fresh impetus to the formation of molecular Bose-Einstein condensates [1]. As several experimental techniques related to cold molecules involve optical excitation either in the formation or detection stages, an accurate need of precise spectroscopic characterization of excited molecular states has arisen. Among alkali-metal diatomic molecules, $\mathrm{NaLi}$ is particularly interested there because it has permanent dipole moment, thus can be manipulated with external electric fields.

Experimental investigations of NaLi molecules, however, encounter two difficult problems. The first one is the difficulty in producing NaLi molecules in gas phase due to a big difference in melting-point temperatures between $\mathrm{Na}$ and $\mathrm{Li}$ bulks. The second one is the spectral overlaps due to a large number of allowed electric dipole transitions between various rovibrational energy levels in electronic states of $\mathrm{NaLi}, \mathrm{Na}_{2}$ and $\mathrm{Li}_{2}$. Up to date, number of studied electronic states is quite limited [2-7]. Even for the low-lying states like as the $2^{1} \Pi$ state only a sole experimental work has been reported [2], but without any information on rotational structure because of low resolution of the measurement.

As suggested by Schawlow's group, the problem for spectroscopic overlaps could be surmounted by using the polarization labelling spectroscopy (PLS) technique [8]. The basic idea of the PLS is to monitor changes in polarization of a weak probe laser beam after passing through a molecular sample, which is placed between two crossed linear polarizers, optically pumped by a strong laser light coming from another laser. When the pump laser is off the molecular sample optically isotropic, thus the probe laser beam is blocked at the 
second polarizer. When the pump beam is on, the pump light depopulates unequally the degenerated sublevels in the lower level of the pumped transition, thus makes molecular sample selectively anisotropic. Therefore, whenever the probe light excites a transition at which shares the lower level (so called "labelled" level) with the pumped transition, the polarization of the probe beam is changed, thus the probe light beam passes through the second polarizer and reaches to the detector.

In this work, we present our PLS measurements for NaLi. To our knowledge, this is the first time the rotational structure of $\mathrm{NaLi}$ in the $2^{1} \Pi$ state has been resolved.

\section{EXPERIMENT}

The PLS arrangement for NaLi is schemed in Fig. 1. We used a homebuilt Littmantype dye laser and a Lumonics 500 dye laser as the probe and pump sources, respectively. Both lasers were pumped synchronously by the LightMachinery Excimer laser. The Lumonics HD 500 dye laser works with Styben 3 and Coumarin. It delivers pulse-energy of ca $5 \mathrm{~mJ}$ with $0.05 \mathrm{~cm}^{-1}$ spectral linewidth. The homebuilt dye, which works with DCM, provides weak pulse energy of $c a 0.05 \mathrm{~mJ}$ with $c a 0.5 \mathrm{~cm}^{-1}$ spectral linewidth. The probe laser wavelength was tuned to excite resonantly transitions in the $2^{1} \Sigma-1^{1} \Sigma$ band. It was measured with a HighFinesse WS-6 wavemeter (Toptica). We also used a $496.5 \mathrm{~nm} \mathrm{Ar}{ }^{+}$ laser line to excite a known probe transition in the $1^{1} \Pi-1^{1} \Sigma^{+}$band. The pump and probe laser beams were directed to propagate almost collinearly together, thus ensuring maximum overlap of the beams inside the sample. The polarization of the pump beam was set to either linear or circular by a removable $\lambda / 4$ wave-plate.

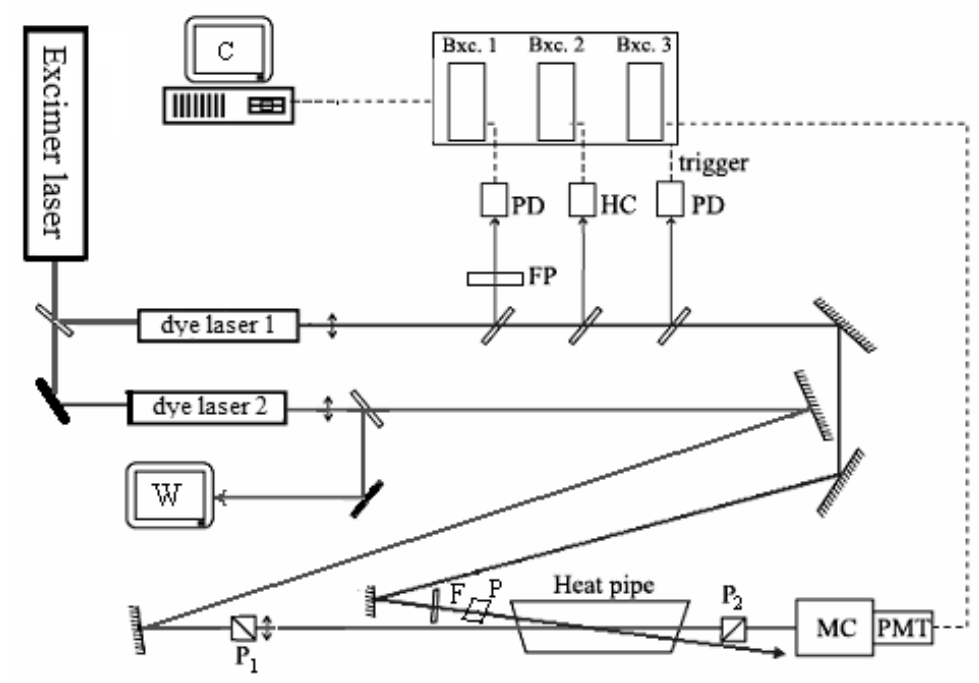

Fig. 1. Experimental setup for V-type PLS: Bxc- boxcar integrator, FP- FabryPerot interferrometer, HC- hollow-cathode lamp, F- $\lambda / 4$ wave plate, MCmonochromator, P- polarizer, PD- photodiode, PMT- photomultiplier tube.

The NaLi molecules were produced in a home-made three-section heat-pipe oven [9]. The heat-pipe is made from a stainless steel pipe which is surrounded by three heaters 
connected to the separated power supplies that allow independent control of the temperature of each heater. At both ends, the pipe is closed with fused silica windows which are fixed by easily demountable clamped flanges. A piece of Li was loaded to the central part of the heat-pipe whereas two pieces of $\mathrm{Na}$ were placed at outer parts. After the oven evacuated, about 4 Torr of Helium was filled into it to serve as buffer gas. Finally, the Li and $\mathrm{Na}$ bulks were heated to temperatures $650^{\circ} \mathrm{C}$ and $390^{\circ} \mathrm{C}$, respectively.

The pump laser was tuned to scan over the region $21500-24000 \mathrm{~cm}^{-1}$ in either linear or circular polarization mode. During the scanning, small parts of the pump laser beam were sent to a Fabry - Perot interferrometer (with FSR $\approx 1 \mathrm{~cm}^{-1}$ and $F=80$ ) and two neon- and argon-filled hollow-cathode lamps. The transmission interference and optogalvanic signals were recorded by two SR 250 boxcar integrators (Stanford Research Systems), as indicated by the notations Bxc 1 and Bxc 2 in figure 1. The interfering fringes and optogalvanic spectrum were used for calibration of PLS spectrum. The probe light passing through the second polarizer is sent to a monochromator and then detected by a photomultiplier tube. The use of monochromator here is to eliminate fluorescence coming from the heat-pipe oven. The polarization signal was recorded at the third SR 250 boxcar (Bxc3), simultaneously with the optogalvanic and interfering transmission signals. Finally, three boxcars were connected to a control computer via a SR 245 computer interface (Stanford Research System).

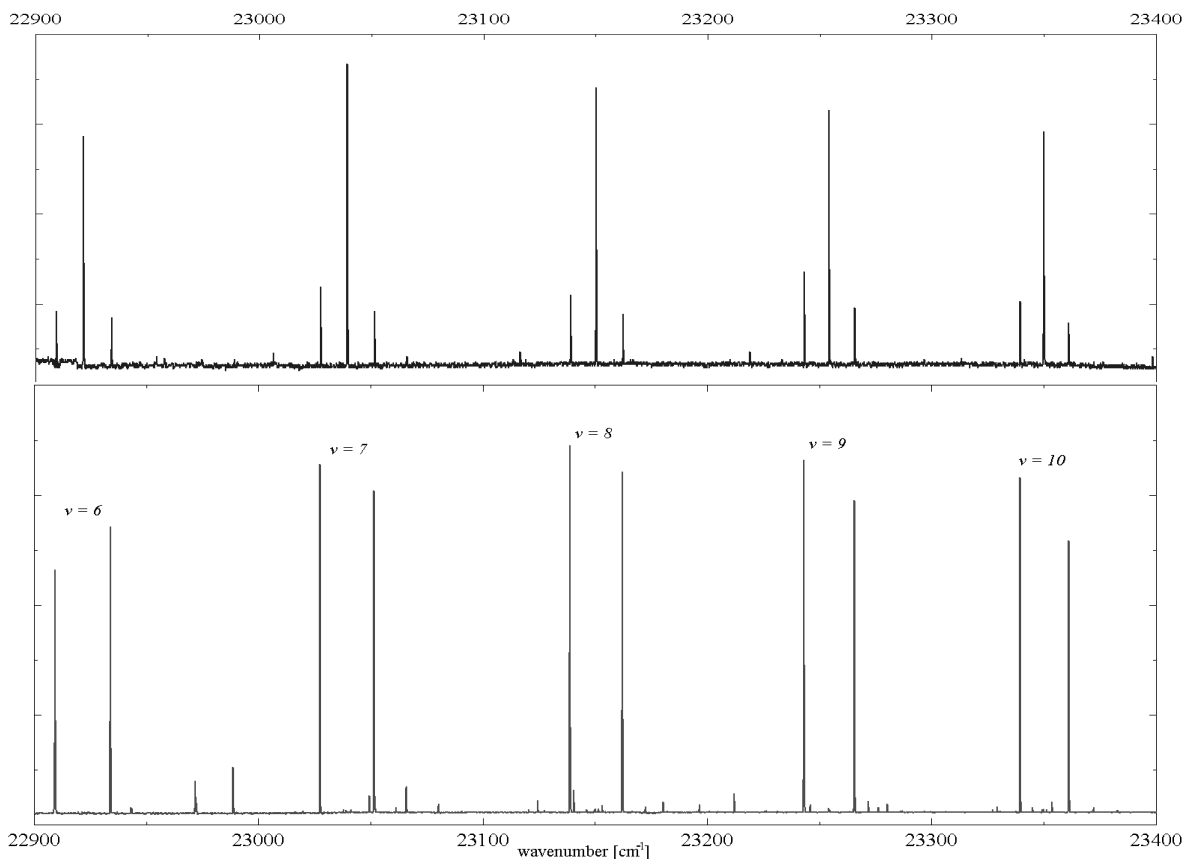

Fig. 2. A fragment of PLS spectrum observed in the case of linear (upper) and circular (down) polarization of the pump beam when probing at $496.5 \mathrm{~nm}$, thus labels level $(0,30)$ in the ground electronic state. The vibration quantum number is indicated in this figure. 
In the scanning spectral region of pump laser wavelength, we performed measurements corresponding to 30 labelled levels. Practically, the efficiency for simplification of PLS spectrum strongly depends on the choice of which probe transition being used. In our work, we calculated probe wavenumbers by using the set of molecular constants determined in [3], and then removed out the wavenumbers of which promote simultaneously transitions in $\mathrm{Na}_{2}$ and/or $\mathrm{Li}_{2}$. We finally optimized the calculated probe wavenumbers by tuning the probe laser to the proper wavelength in the way of having strongest PLS signal. The final probe wavenumbers and the corresponding labelled levels are listed in Table 1. It is noted that, since the broaden linewidth of the probe laser $\left(c a .0 .5 \mathrm{~cm}^{-1}\right)$ so in some cases it excites simultaneously two labelling levels.

Table 1. The probe laser lines and the corresponding labelled levels used in this work

\begin{tabular}{cc|cc}
\hline \hline Laser line & Labelled level $(\nu, J)$ & Laser line & Labelled level $(\nu, J)$ \\
\hline \hline $15433.42 \mathrm{~cm}^{-1}$ & $(0,3)$ & $15544.80 \mathrm{~cm}^{-1}$ & $(0,21)$ \\
\hline $15083.76 \mathrm{~cm}^{-1}$ & $(0,5)$ & $15463.80 \mathrm{~cm}^{-1}$ & $(1,21),(1,56)$ \\
\hline $15435.14 \mathrm{~cm}^{-1}$ & $(0,6)$ & $15390.05 \mathrm{~cm}^{-1}$ & $(0,22)$ \\
\hline $15074.04 \mathrm{~cm}^{-1}$ & $(0,7)$ & $15385.30 \mathrm{~cm}^{-1}$ & $(0,23)$ \\
\hline $15594.71 \mathrm{~cm}^{-1}$ & $(0,9)$ & $15427.50 \mathrm{~cm}^{-1}$ & $(0,24)$ \\
\hline $15425.10 \mathrm{~cm}^{-1}$ & $(0,12)$ & $15521.20 \mathrm{~cm}^{-1}$ & $(0,25),(1,10)$ \\
\hline $15401.00 \mathrm{~cm}^{-1}$ & $(0,15)$ & $15440.20 \mathrm{~cm}^{-1}$ & $(1,25)$ \\
\hline $15565.40 \mathrm{~cm}^{-1}$ & $(0,17)$ & $15493.50 \mathrm{~cm}^{-1}$ & $(0,29),(0,47)$ \\
\hline $15484.20 \mathrm{~cm}^{-1}$ & $(1,17)$ & $496.5 \mathrm{~nm}^{-1}$ & $(0,30)$ \\
\hline $15560.60 \mathrm{~cm}^{-1}$ & $(0,18),(0,45)$ & $15357.16 \mathrm{~cm}^{-1}$ & $(2,30)$ \\
\hline $15402.96 \mathrm{~cm}^{-1}$ & $(0,19)$ & $15303.60 \mathrm{~cm}^{-1}$ & $(2,33),(1,46)$ \\
\hline $15303.60 \mathrm{~cm}^{-1}$ & $(1,19)$ & $15357.16 \mathrm{~cm}^{-1}$ & $(1,36)$ \\
\hline $15398.88 \mathrm{~cm}^{-1}$ & $(0,20)$ & \multicolumn{2}{c}{} \\
\hline \hline
\end{tabular}

\section{RESULTS AND DISCUSSIONS}

In our experiment, at the beginning we used $496.5 \mathrm{~nm} \mathrm{Ar}^{+}$laser line, which excites the level $(0,30)$ in the ground state, as the probe and then performed PLS measurements with circular and linear polarization modes of the pump beam. The initial choice of such probe laser wavelength was the reasonable way to check if concentration of NaLi molecules produced significantly or not. The PLS spectra observed in the two polarization modes, on the other hand, were the evidence to determine symmetry of the observed state. Indeed, we observed P, Q and R branches in linear polarization mode whereas only P- and R branches appeared in the circular one (Fig. 2). Such a feature shows that the observed PLS spectra associate to a ${ }^{1} \Pi$ symmetric electronic state [10]. Furthermore, we could attribute the state as the $2^{1} \Pi$ by using the atomic asymptotic [11].

Knowing the symmetry of the electronic state, we then performed measurements at the linear polarization mode with the remained labelling levels. Figure 3 shows a part of PLS spectrum observed with $15594.71 \mathrm{~cm}^{-1}$ probe laser line, which labels the level $(v=$ 
$0, J=9)$ in the ground electronic state. For this labeling level we observed the vibration progression up to vibration quantum number $v=15$. By comparing wavenumber of the last spectral line in the last triplet with the atomic asymptotic [11] we see that the spectral line positions are near to the dissociation limit. Such obtained data are important to investigate long-range interactions between $\mathrm{Na}$ and $\mathrm{Li}$.

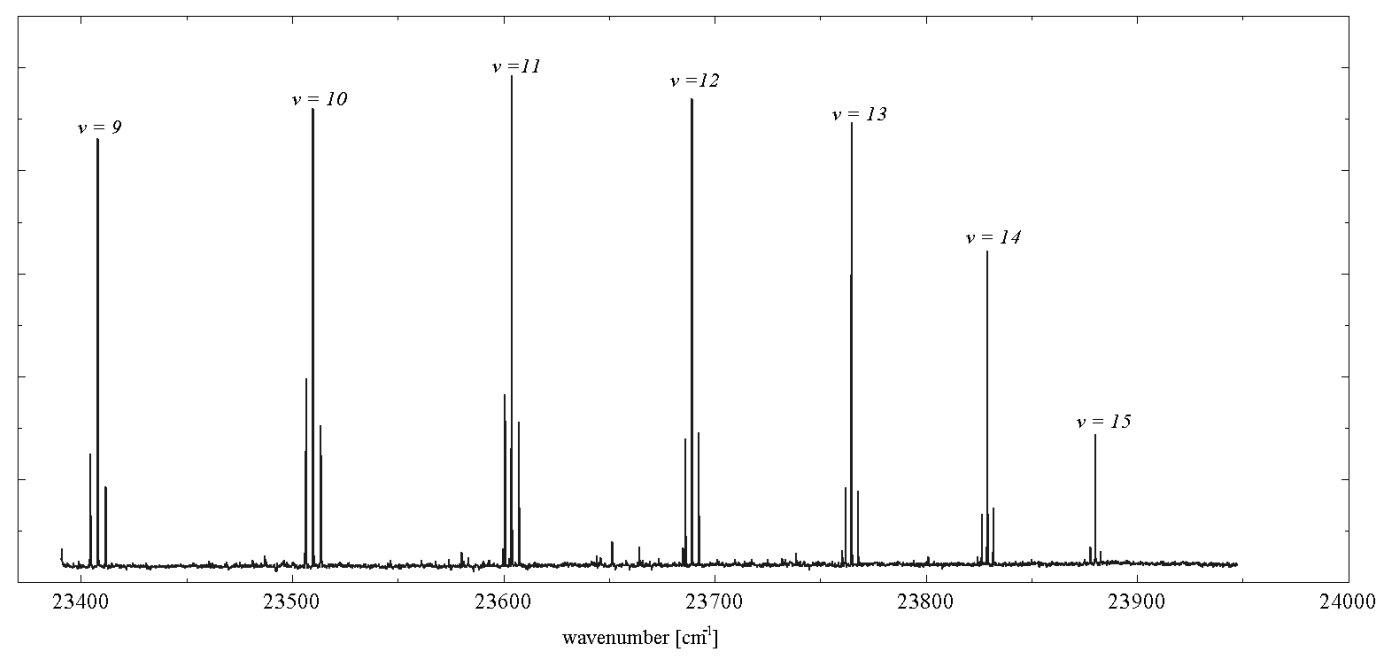

Fig. 3. A fragment of PLS spectrum near the dissociation limit observed in the case of linear pump polarization and $15594.71 \mathrm{~cm}^{-1}$ probe wavenumber, which labels level $(0,9)$ in the ground state. The vibration quantum number is indicated in this figure.

It should be mentioned here that, the investigated spectral region covers also the spectra of $\mathrm{Na}_{2}$ and $\mathrm{Li}_{2}$. However, due to the proper choice of probe wavelength for labelling, the registered PLS spectra were almost of NaLi. Finally, our measurements with 30 labelled levels giving 814 spectral lines associate to the $2{ }^{1} \Pi$ state. Details of spectroscopic characterization for the observation will be reported [12].

\section{CONCLUSION}

The polarization labelling spectroscopy technique has been employed successfully to explore NaLi molecule at the $2^{1} \Pi$ electronic state, in which the rotational structure has been resolved for the first time. It has been proven to be an efficient technique. Although the polarization spectra are Doppler-limited, they are greatly clarified in comparison to normal absorption spectra due to the labelling process. By proper choice for probe wavelength it could be possible to eliminate unwanted transitions, thus making spectroscopic analyzing procedure less effort.

\section{ACKNOWLEDGMENTS}

The financial supports from the Vietnams National Foundation for Science and Technology Development (NAFOSTED) under the project code 103.06.110.09 and from 
Ministry of Science and Technology under the project code 18/2010/HD-NDT are acknowledged. We would like to thank Professor Jastrzebski for supporting our PLS measurements in his lab, and Dr J. Szczepkowski for experimental assistance.

\section{REFERENCES}

[1] S. Jochim et al., 302 (2003) 2101.

[2] M. M. Kappe, K. O. Marti, P. Radi, M. Schäp, and E. Scgumacher, Chem. Phys. Lett. 107 (1984) 6.

[3] C. E. Fellows, J. Chem. Phys. 94 (1991) 5855; J. Mol. Spectrosc 136 (1989) 369.

[4] C. E. Fellows, J.Vergés, and C. Amiot, J. Chem. Phys. 93 (1990) 6281-6290.

[5] W. Jastrzebski, P. Kowalczyk, R. Nadyak, and A. Pashov, Spectrochim. Acta, Part A, 58A (2002) 2193.

[6] I. D. Petsalakis et al., Chem. Phys. 362 (1999) 130.

[7] Nguyen Huy Bang et. al., J. Mol. Spectr. 233 (2005) 290; Optica. Applicata 36 (4) (2006) 499; Chem. Phys. Lett. 440 (2007) 199; Opt. Mat. 31 (2009) 527; J. Chem. Phys. 130 (2009) 124307.

[8] R. Teets, R. Feinberg, T. W. Hansch, and A. L. Schawlow, Phys. Rev. Lett. 37 (1976) 683.

[9] V. Bednarska, Jackowska, W. Jastrzebski, and P. Kowalczyk, Meas. Sci. Technol. 7 (1996) 1921.

[10] R. Ferber, W. Jastrzebski, and P. Kowalczyk, J. Quant. Spectrosc. Radiat 58 (1997) 53.

[11] A. A. Radzig and P. M. Smirnov, Reference Data on Atoms, Molecules and Ions, Springer, Berlin, 1985.

[12] Nguyen Huy Bang et al., to be published in Chem. Phys. Lett. (2011)

Received 29 December 2010. 